\title{
Prompt Photon physics in ALICE: Gamma-hadron and Gamma-Jet correlations
}

\author{
Gustavo Conesa Balbastre*† \\ INFN-Frascati Italy \\ E-mail: Gustavo.Conesa.Balbastre@cern.ch
}

Prompt photons and light neutral-mesons will be detected and identified in the ALICE experiment at LHC with the PHOS detector and with the EMCal detector, whereas charged particles will be detected and identified by the central tracking system. We examine in this proceedings the possibility to do photon-tagged correlations in order to study how the dense partonic medium produced in heavy-ion collisions affects fragmentation of high energy quarks and gluons. Methods to identify prompt photons and to distinguish them against the background of decay and fragmentation photons are discussed.

High-pT physics at LHC

March 23-27, 2007

University of Jyvaskyla, Jyväskylä, Finland

\footnotetext{
* Speaker.

${ }^{\dagger}$ Thanks to F. Arleo, H. Delagrange, J. Díaz, Y.V. Kharlov and Y. Schutz.
} 


\section{Introduction}

The experimental study of hadron jets at LHC is expected to provide decisive data for understanding the properties of the quark gluon plasma (QGP) formed in ultra-relativistic nucleusnucleus collisions [1]. Hadron jets are generated by the hadronization of final-state partons with high transverse momentum $\left(p_{T}\right)$ scattered in primary collisions. Bjorken suggested more than 20 years ago that partons propagating through a nuclear medium suffer an energy loss which is strongly dependent on the color charge density of the medium [2]. Medium modification manifests as a modification of the energy spectrum of jet hadrons, which is known as the jet quenching effect. This effect has indeed been observed in central Au-Au collisions at $\sqrt{s_{N N}}=130$ and $200 \mathrm{GeV}$ in measurements of high $p_{T}$ charged and neutral hadrons $\left(p_{T} \sim 2-15 \mathrm{GeV} / c\right)$ by various RHIC experiments [3,4] in which the yields of inclusive charged hadrons and $\pi^{o}$ mesons are suppressed by as much as a factor 5 , independent of their $p_{T}$ value, compared to the properly scaled $p p, d$-Au and peripheral $\mathrm{Au}-\mathrm{Au}$ yields.

The ALICE experiment will extend these studies to much higher $\sqrt{s}$. Due to the larger cross sections of hard processes at LHC compared to RHIC, jets will be abundantly produced at LHC $\left(2 \times 10^{6}\right.$ jets with $p_{T}>100 \mathrm{GeV} / c$ per year in the ALICE acceptance are expected) allowing inclusive and exclusive jet measurements. In particular, jet characteristics (jet shape, jet heating, fragmentation function...) could be measured in order to study the energy distribution of jets [5]. To carry out these studies, the identification of jets and the accurate measurement of the jet energy before and after quenching is required. There are two very attractive methods to perform these measurements, tagging high energy hadrons or jets with prompt photons emitted opposite to the hadrons or jet direction $[6,7]$. A prompt photon is produced directly in the hard subprocess, back-to-back to a quark which loses some energy (through medium-induced gluon radiation) before eventually fragmenting into a leading pion. The dominant elementary processes, leadingorder, which produce such events are $g+q \rightarrow \gamma+q$ (Compton) and $q+\bar{q} \rightarrow \gamma+g$ (annihilation), although recent theoretical studies show that high order bremsstrahlung processes also contribute significantly to the photon yield below $50 \mathrm{GeV} / c$ [6]. Photons emerge almost unaltered from a dense medium and provide a measurement of the original transverse momentum and azimuth of the parton emitted in the opposite direction. Medium effects could be identified from the behavior of the fragmentation function, i.e., the distribution of the jet energy among the jet constituents, rather than from the total jet energy.

In order to study the modifications of the medium on the jet, we may avoid making the full jet reconstruction, we can just study the correlation between the prompt photons and the high energy hadrons, opposite to the photon, that belong to the jet. This has been investigated in detail at RHIC and LHC energies [8]. Because of momentum conservation, the $\gamma-\pi^{0}$ momentum imbalance variable,

$$
z_{\gamma \pi} \equiv-\frac{\mathbf{p}_{\perp \pi} \cdot \mathbf{p}_{\perp \gamma}}{\left|\mathbf{p}_{\perp \gamma}\right|^{2}}
$$

reduces to the fragmentation variable, $z_{\gamma \pi}=z$, in this leading-order (LO) kinematics. Therefore, there should be a clear connection between the experimentally accessible momentum-imbalance distributions and the theoretical quark fragmentation function into a pion. This reasoning is of course too simplistic, since the photon can itself be produced by the collinear fragmentation of 
a leading parton to fragmentation and decay photons. In this case, there is no simple correlation between the pion and the photon momenta, and the correspondence between experimental and theoretical fragmentation function is lost. As we shall see, this is precisely the most important "background" channel we would like to reduce with appropriate kinematic cuts. Moreover, higherorder corrections as well as initial- and final-state soft gluon radiation could complicate somehow the picture.

In ALICE, photons will be detected by the PHOton Spectrometer (PHOS) which enables to measure with high precision their 4-momentum, although, only in a limited acceptance [9], and also by the ElectroMagnetic Calorimeter (EMCal) [10, 11], about seven times larger but with slightly worse resolution and coarser granularity (design still under discussion). The identification power of prompt photons is limited by the background created by decay photons (mainly from $\pi^{0}$ decay). The identification of photon-jet events in ALICE is optimal for photons with energy larger than $20 \mathrm{GeV}$. Below this value, decay and prompt photons cannot be efficiently separated on an event by event basis. In the present article, we discuss the feasibility of identifying prompt photons in $p p$ and heavy-ion collisions by analysis of topological characteristics of the shower and isolation criteria (Sec. 3). In addition, we discuss algorithms for identifying photon-jet (Sec. 5) and photon-hadron (Sec. 5) events for reconstructing hadron jet features. A detailed description of the work discussed here can be found in Refs. [12, 13].

\section{Event simulation and main reconstruction features}

Acceptances and energy and position resolutions for all the detectors involved in this study (PHOS and $\mathrm{EMCal}^{1}$ for photons and $\mathrm{TPC}^{2}$ for charged particles) are reported in Tab. 1. A full description of the ALICE detector is given in Ref. [14].

\begin{tabular}{cccccc}
\hline Detector & $|\eta|$ & $\phi_{\min }$ & $\phi_{\max }$ & $\sigma_{E} / E(\%)$ & $\sigma_{p o s}$ \\
\hline \hline PHOS & 0.12 & $220^{\circ}$ & $320^{\circ}$ & $1-1.5$ & $0.8-2.5 \mathrm{~cm}$ \\
TPC & 0.7 & $0^{\circ}$ & $360^{\circ}$ & 2 & $1.1^{\circ}$ \\
EMCal & 0.7 & $60^{\circ}$ & $180^{\circ}$ & & \\
\hline
\end{tabular}

Table 1: TPC, EMCal and PHOS detector acceptances, and energy and position resolutions. The real TPC $\eta$ acceptance is larger $(|\eta|<0.9)$, but we selected this lower value to ensure good track matching. The EMCal performance is still under investigation, we assumed in our study same response as PHOS. Azimuthal angles are given in the ALICE global reference system.

We assume that prompt photon production arises from $\gamma$-jet events in the leading order of the Standard Model, comprising Compton and annihilation processes. These processes were simulated with the event generator PYTHIA 6.203 [15] for $p p$ collisions at $\sqrt{s}=5.5 \mathrm{TeV}$. The default parton distribution function, GRV 94L [16], was used. Events were generated in the energy range $20<p_{T}<100 \mathrm{GeV} / c$. In order to enrich the sample with events within the PHOS acceptance,

\footnotetext{
${ }^{1}$ ElectroMagnetic CALorimeter final design still under discussion, geometrical acceptance values have changed since this study was done.

${ }^{2}$ Time Projection Chamber.
} 
we restricted the prompt photon pseudorapidity range to $\left|\eta_{\gamma}\right|<0.2$ and the azimuthal aperture to $200^{\circ}<\phi_{\gamma}<340^{\circ}$ in the event generation.

Events with two jets in the final state, called jet-jet events, are a significant source of background. They were simulated by hard QCD $2 \rightarrow 2$ processes in the leading pQCD order. These processes contribute to the background through hard $\pi^{0}$-mesons which decay photons may be detected in PHOS as single electromagnetic showers and which may mimic prompt photons. To simulate a continuous $p_{T}$-spectrum of $\pi^{0}$-mesons from 20 to $100 \mathrm{GeV} / c$, we generated hard QCD processes in the $p_{T}$ range from 30 to $300 \mathrm{GeV} / c$. The generation of hard QCD processes was restricted to $\left|y_{\text {parton }}\right|<0.2$ and to $\left|\eta_{\text {jet }}\right|<0.15$, without any azimuthal angle limitation. This more severe restriction in rapidity compared to the $\gamma$-jet case was imposed to enrich the fraction of events with detectable $\pi^{o}$-mesons.

$\mathrm{Pb}-\mathrm{Pb}$ collisions were simulated by merging $p p$ collisions generated by PYTHIA with heavyion events produced by the HIJING 1.36 [17] event generator for $\mathrm{Pb}-\mathrm{Pb}$ collisions at $\sqrt{s_{N N}}=$ $5.5 \mathrm{~A} \mathrm{TeV}$ and impact parameter $b<2 \mathrm{fm}$.

In this study, a full-fledged Monte Carlo simulation of the transport of particles in PHOS was carried out. To reduce computing time, we applied a fast reconstruction method for particles detected in the TPC and EMCal [12]. The response of EMCal was assumed, as a first approximation, identical to that of PHOS.

\subsection{Expected experimental rates}

The $p_{T}$ distributions, $N\left(p_{T}\right)$, obtained from simulations were normalized to the number of events expected in a standard LHC running year by,

$$
N\left(p_{T}\right)=\sigma_{A A}\left(p_{T}\right) \cdot \mathscr{L} \cdot t
$$

\begin{tabular}{ccc}
\hline Collision & $\mathscr{L}\left(\mathrm{cm}^{-2} \mathrm{~s}^{-1}\right)$ & $\mathrm{t}(\mathrm{s})$ \\
\hline \hline$p p$ & $10^{30}$ & $10^{7}$ \\
$\mathrm{~Pb}-\mathrm{Pb}$ & $5 \times 10^{26}$ & $10^{6}$ \\
\hline
\end{tabular}

Table 2: Beam luminosity and running time for the ALICE experiment during a standard year of running at LHC.

where $\mathscr{L}$ and $t$ are the luminosity and the experiment running time reported in Tab. 2. The cross section for $p p$ collisions, $\sigma_{p p}$, was obtained from PYTHIA and the cross section for $\mathrm{Pb}-\mathrm{Pb}$ collisions, $\sigma_{A A}$, was calculated by scaling $\sigma_{p p}$ with the "binary scaling" equation,

$$
\left(\frac{d^{2} \sigma_{A A}}{d p_{T} d y}\right)_{C}=\left\langle T_{A A}\right\rangle_{C} \cdot \sigma_{A A}^{g e o} \cdot f_{C} \cdot \frac{d^{2} \sigma_{p p}}{d p_{T} d y}
$$

where $\left\langle T_{A A}\right\rangle_{C}$ is the mean nuclear overlap function for the corresponding centrality class $C, f_{C}$ the centrality factor and $\sigma_{A A}^{g e o}=7745 \mathrm{mb}$ the geometrical cross section given by Eq. (133) of [18]. The resulting spectra for $\mathrm{Pb}-\mathrm{Pb}$ collisions at $5.5 \mathrm{~A} \mathrm{TeV}$, for minimum bias conditions, are shown in Fig. 1, for which $f_{C}=1$ and $\left\langle T_{A A}\right\rangle_{C}=5.58$ ( 0.05 and 26.0 for 5\% centrality, respectively).

\section{Prompt photon identification: Isolation Cut Method}

Two different procedures were applied to select prompt photons from inclusive photons, which include bremsstrahlung and decay photons from jet-jet events: the Shower Shape Analysis (SSA) [9, 19], and the Isolation Cut Method (ICM). In the former method, photons were identified by analyzing the shape of the shower in PHOS, and in the latter method, photons were tagged and identified as prompt if they appear isolated, i.e., without charged particles in their vicinity. 
Photon spectra from $\gamma$-jet and jet-jet events were identified with the PHOS SSA analysis. The shower generated in the PHOS calorimeter by a particle can be characterized by several parameters which define the shower shape. Usually, they are taken as the length of the principal axes of the shower surface, $\lambda_{0}$ and $\lambda_{1}$, the shower lateral dispersion, the core energy, the sphericity defined as $\left(\lambda_{0}-\lambda_{1}\right) /\left(\lambda_{0}+\lambda_{1}\right)$, the maximal energy fraction deposited in one single crystal and the shower multiplicity [19]. These parameters are found to be correlated to a large extent. To select a smaller number of parameters conveying the maximal information about the shower shape, we uncorrelated the above parameters through a principal component analysis in which these seven parameters are transformed into new seven uncorrelated parameters given by the eigenvectors of the covariance matrix. We found that a good description of the shower shape is obtained when only the two most significant parameters, corresponding to the largest eigenvalues, are kept. These two principal components are found to be distributed in a Gaussian way for large samples of photon showers. We defined low, medium and high purity photons as those within three, two and one standard deviations,

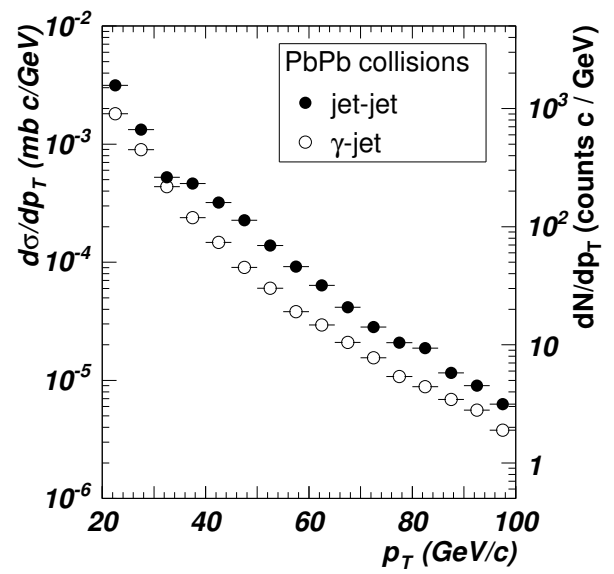

Figure 1: $\quad$ Spectra of prompt photons $(\gamma$-jet events, $\circ$ ) and $\gamma$-like (single and overlapped photons from jet-jet events, •) detected in $\mathrm{PHOS}$, for minimum bias $\mathrm{Pb}-\mathrm{Pb}$ collisions at $\sqrt{s_{N N}}=5.5 \mathrm{~A} \mathrm{TeV}$. Differential cross sections are given on the left $y$-axis of the plots and the expected number of particles on the right $y$-axis. Both quantities are determined for a standard LHC running year. respectively, of the mean of their Gaussian distributions. For medium purity level, the prompt photon identification efficiency is about $85 \%$ for $p p$ collisions and about $75 \%$ for $\mathrm{Pb}-\mathrm{Pb}$ collisions. The misidentification probability of background events as prompt photons ranges, as a function of $p_{T}$, from 0 to $40 \%$ for one-cluster neutral pions ${ }^{3}$ and medium purity identification, and from 0 to $15 \%$ for hadrons. The remaining $\pi^{0}$ background has a contribution similar to the prompt photon signal. Requiring higher purity photons, improved the background rejection at the cost of an important reduction of the identification efficiency. To keep the identification efficiency to an acceptable value while achieving a good background rejection, additional identification procedures are required.

Since prompt photons are produced in parton collisions in which the final state photon and parton are emitted in opposite directions, no hadron belonging to the parton jet fly in the same direction as the photon ${ }^{4}$. However, the underlying event generated by the heavy-ion collision may perturb this ideal geometrical scheme. We have developed two isolation algorithms, both based on the search for hadrons inside a cone centered around the direction $\left(\eta_{0}, \phi_{0}\right)$ of high- $p_{T}$ photon

\footnotetext{
${ }^{3}$ An energetic $\pi^{o}, E>30 \mathrm{GeV}$, decays into two photons with a too small opening angle to be separated in PHOS, generating in this way a single cluster.

${ }^{4}$ This is not true for next to leading order processes like bremsstrahlung. However, PYTHIA predicts that such processes are suppressed compared to $\pi^{o}$ production. This statement might have to be revised according to recent studies[6], which suggest that at high $p_{T}$ the bremsstrahlung could be a dominant process $\left(p_{T}<50 \mathrm{GeV} / c\right)$.
} 
candidates $\left(p_{T}>20 \mathrm{GeV} / c\right)$ identified by the SSA. The cone size is given by

$$
R=\sqrt{\left(\phi_{0}-\phi\right)^{2}+\left(\eta_{0}-\eta\right)^{2}} .
$$

For $\gamma$-jet events in $p p$ collisions, there is almost no particle inside the cone, independently of the energy of the prompt photon but for jet-jet events a clear dependence of the particle multiplicity inside the cone on the jet energy is found. Thus, the $p_{T}$ distribution of particles inside a cone around a photon candidate can be used to distinguish between $\gamma$-jet and jet-jet events. Following this idea, we have developed two different selection criteria to decide if a photon candidate is isolated and can be accepted as a prompt photon:

1. There is no hadron with $p_{T}$ above a given threshold $p_{T}^{\text {th }}$ in the cone.

2. The sum of the transverse momentum of all hadrons inside the cone, $\Sigma_{p_{T}}$, must be smaller than a given threshold.

After an exhaustive analysis we found the following optimal parameters for prompt photon identification:

- In the case of $p p$ collisions, a $\gamma$-jet identification probability of $100 \%$ and a jet-jet misidentification probability of $3 \%$ was obtained with $R=0.2$ and $\Sigma_{p_{T}}<0.7 \mathrm{GeV} / c$.

- In the case of $\mathrm{Pb}-\mathrm{Pb}$ collisions, a $\gamma$-jet identification probability of $50 \%$ and a jet-jet misidentification probability of $7 \%$ was obtained with $R=0.2$ and $p_{T}^{t h}=2 \mathrm{GeV} / c$. The resultant prompt photon spectra (Fig. 2) indicate that a sufficient background reduction was attained.

We conclude that a sufficient background rejection is achieved by the ICM for $p p$ and $\mathrm{Pb}-\mathrm{Pb}$ collisions. In the case that a quenching factor of 5 , as reported by RHIC [20], exists at LHC energies, the signal to background ratio would increase from 4 to 20 .

\subsection{Final prompt photon spectrum}

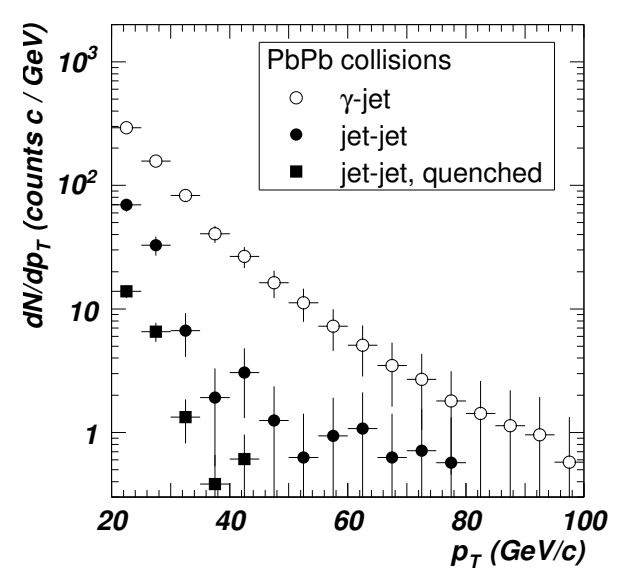

Figure 2: Spectra with statistical errors of events identified as prompt photons in ALICE during a LHC running year by medium purity SSA and ICM $(R=0.2$, $p_{T}^{\text {th }}=2 \mathrm{GeV} / \mathrm{c}$ ) methods, due to jet-jet $(\bullet)$ and $\gamma$-jet events (o), for $\mathrm{Pb}$ - $\mathrm{Pb}$ collisions at $5.5 \mathrm{~A} \mathrm{TeV}$. The spectrum obtained with a quenching factor of 5 for jet-jet events is also shown (-).

We have constructed the prompt photon spectrum with the corresponding statistical and systematic errors expected to be measured during one LHC running period. We obtained the total identified prompt photon spectrum $N_{\gamma}^{i d}$, as the addition of the identified prompt photon spectrum from $\gamma$-jet events to the background spectrum due to jet-jet events. From the known identification probabilities, we can reconstruct the original prompt photon spectrum as follows: let $N_{\gamma}$ be the original prompt photon spectrum, $N_{\pi^{o}}$ the original $\pi^{o}$ spectrum, $N_{h}$ the original hadron spectrum, 
$\varepsilon_{i}^{i d}$ the identification probability of particle $i$ as a photon by SSA and $\varepsilon_{i}^{i c}$ the identification probability of particle $i$ as prompt photon by ICM, where $i$ can be a photon, a one-cluster $\pi^{0}$ or any other hadron. We can write

$$
N_{\gamma}^{i d}=N_{\gamma} \varepsilon_{\gamma}^{i d} \varepsilon_{\gamma}^{i c}+N_{\pi^{o}} \varepsilon_{\pi^{o}}^{i d} \varepsilon_{\pi^{o}}^{i c}+N_{h} \varepsilon_{h}^{i d} \varepsilon_{h}^{i c}=\zeta N_{\gamma}
$$

As discussed in the previous sections, we deduced each of the factors needed above (the ICM misidentification probabilities for $\pi^{o}$ and hadrons are almost identical) and calculated the correction factor $\zeta$ for the various sets of identification criteria (purity levels, cone sizes and $p_{T}$ thresholds). The systematic error of the particle identification methods (PID) was calculated as the dispersion of the corrected spectra obtained by the different identification criteria. This systematic error was added quadratically to the average background spectra in order to obtain the total systematic error. The statistical error was calculated from the photon statistics as $\sqrt{N_{\gamma}^{i d}}$. The final spectrum of identified photons and its comparison with the original spectra are shown in Fig. 3. If the assumption that hadrons are quenched by a factor 5 is made, the systematic error is significantly reduced.
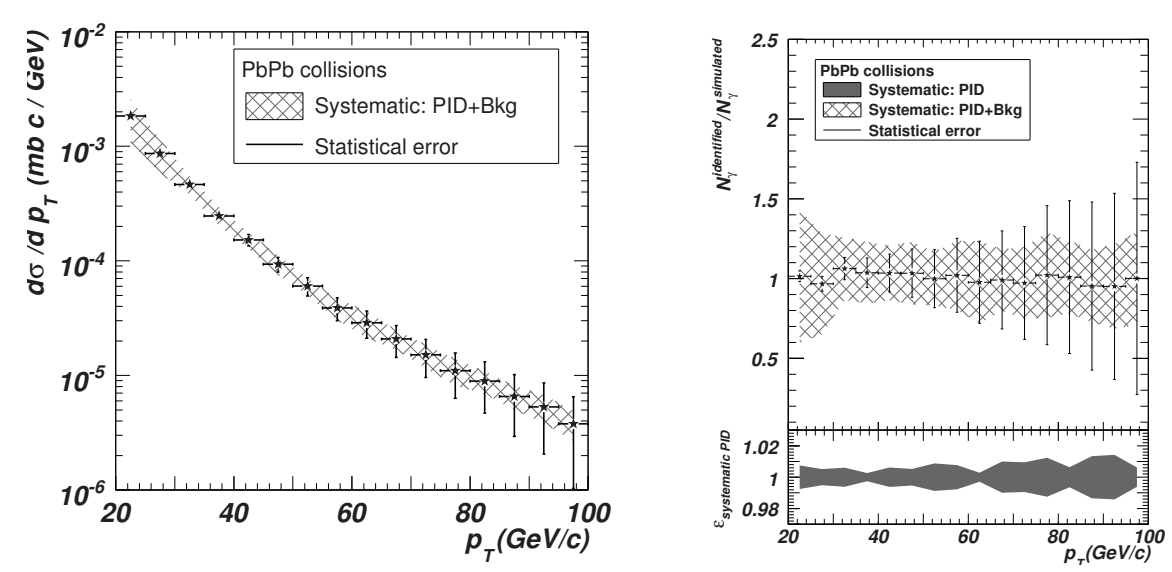

Figure 3: Left frame: Simulated final prompt photon spectrum measured in ALICE during a LHC running year with statistical and systematic (background + PID) errors in PbPb collisions. Right frame: Ratio of the corrected prompt photon spectrum to the original simulated spectrum in $\mathrm{PbPb}$ collisions. The upper frame shows the ratio with the statistical and systematic bands due to background plus PID and the lower frame shows just the systematic error band due to the PID.

\section{Photon-tagged jets}

We developed an algorithm to tag jets by prompt photons. Two different experimental configurations were considered : i) Charged particles are detected in the central tracking system (TPC) and neutral particles in EMCal; this configuration is labeled as TPC+EMCal; ii) Only the central tracking system is available and consequently only charged particles can be detected; this configuration is labeled as TPC. The steps of the algorithm are:

1. Search in each event for the most energetic prompt photon identified by PHOS. 
2. Search for the jet leading particle ${ }^{5}$ (the charged hadron or neutral pion with the highest $p_{T}$ value), detected by the central tracking system or EMCal, and emitted almost opposite to the photon in azimuthal angle, i.e., with $\Delta \phi$ close to $180^{\circ}, 0.9 \pi<\Delta \phi<1.1 \pi$. An additional condition to be satisfied by the leading particle is that its $p_{T}$ value must be at least the $10 \%$ of the photon energy.

3. Reconstruct the jet as the ensemble of all particles contained inside a cone defined by Eq. (3.1) with axis aligned along the leading particle direction. We have taken here $R=0.3$, and the particle $p_{T}$ threshold as $0.5 \mathrm{GeV} / c$.

4. Finally, the event is identified as a photon-jet pair if the ratio of the reconstructed transvers momentum of the jet, $p_{T, j}$, and the prompt photon energy differs by less than a given value. In the case of $p p$ collisions, a photon-jet event observed in the setup including EMCal was well identified if the ratio $p_{T, j} / E_{\gamma}$ is close to one, as displayed in Fig. 4 for $40 \mathrm{GeV}$ jets. In the case of $\mathrm{Pb}-\mathrm{Pb}$ collisions, the background is very important and the $p_{T, j} / E_{\gamma}$ distributions are wide and peak at values larger than one. We required in this case a higher particle momentum threshold, $p_{T}>2 \mathrm{GeV} / c$, to calculate the energy of the jet. We took two different values for the lower $p_{T, j} / E_{\gamma}$ limit depending on the experimental setup: 0.3 for the configuration without EMCal and 0.8 for the configuration with EMCal.

The jet reconstruction algorithm failed for jets with $p_{T}<10 \mathrm{GeV} / c$ because the ratio $p_{T, j} / E_{\gamma}$ suffers from large fluctuations in this case. Therefore, we excluded these jets from our investigation.

We studied the jet selection efficiency, defined as the ratio of the number of identified $\gamma$-tagged jets to the number of prompt photons found in PHOS. The efficiency of the configuration without EMCal is 40-50\% which is larger than the efficiency for the configuration with EMCal (30 \%) due to the following points: i) the wider selection angular range for the configuration without EMCal (which is also associated to a lower identification quality); and ii) the requirement that jets measured in the configuration with EMCal fall completely into the EMCal acceptance which is smaller than that of the central tracking system. We applied also the $\gamma$-jet algorithm to jet-jet events in order to estimate the contamination due to these events. If no prompt photon identification is performed in PHOS,

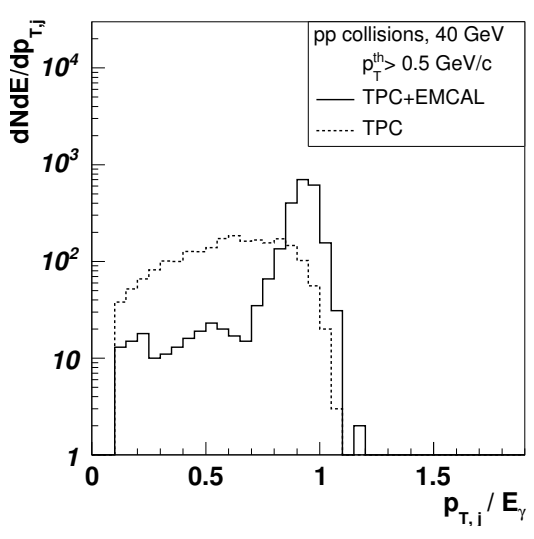

Figure 4: Simulated jet distribution as a function of the ratio $p_{T, j} / E_{\gamma}$ for $40 \mathrm{GeV} \gamma$ jet events in the case of $p p$ collisions. A jet cone of $R=0.3$ and a jet particle threshold of $p_{T}>0.5 \mathrm{GeV} / c$ were assumed. Results for the setups without EMCal (dashed line) and with EMCal (solid line) are given. only about $10 \%$ of the events were accepted in the setup with EMCal but the value raises to $40-50 \%$ in the absence of EMCal. Similar results were obtained for both $p p$ and $\mathrm{Pb}-\mathrm{Pb}$ collisions.

\footnotetext{
${ }^{5}$ A significant proportion of the jet energy (in average $40 \%$ ) is always carried by a few particles.
} 


\subsection{Fragmentation functions}

A satisfactory observable for studying quantitatively the interaction of jets with the medium is the phase space distribution of hadrons from jet fragmentation [5], which is called the jet fragmentation function. The fragmentation function is the distribution of charged hadrons within jets as a function of the variable $z$, defined for hard processes with a $\gamma$-jet pair in the final state as $z=p_{T} / E_{\gamma}$ where $p_{T}$ is the transverse momentum of the hadron from the fragmenting jet. Simulations of jet fragmentation functions expected to be measured in a standard year of LHC running for both $p p$ and $\mathrm{Pb}-\mathrm{Pb}$ collisions, were carried out. Identified $\gamma$-jet events in the energy range from 20 to $100 \mathrm{GeV}$ were considered. The fragmentation functions obtained for jet-jet events misidentified as $\gamma$-jet events were also studied. Figure 5-left shows the fragmentation function, not normalized by the number of collisions, for $\mathrm{Pb}-\mathrm{Pb}$ collisions. The following conclusions are drawn:

- For $p p$ collisions, a signal ( $\gamma$-jet) to background (jet-jet) ratio of about 20 in the configuration without EMCal and almost a $100 \%$ background rejection for the setup with EMCal was obtained. Prompt photon identification reduces the statistics of $\gamma$-jet by a $15 \%$.

- In the case of $\mathrm{Pb}-\mathrm{Pb}$ collisions, the contribution from the heavy-ion collision (HIC) underlying event has been eliminated statistically in the final distributions by subtracting a pseudofragmentation function calculated outside the cone of the leading particle. The final signal to background ratio obtained is about 4 in the case without EMCal and rises to about 10 with EMCal. Prompt photon identification reduces the statistics of $\gamma$-jet events by a $60 \%$.

To evaluate the sensitivity of photon-tagged jet fragmentation functions to nuclear medium modifications, we have calculated the nuclear modification factor $R_{F F}$ which is defined as the ratio of the fragmentation function measured in $A A$ collisions to the fragmentation function measured in $p p$ collisions. This factor should be equal to one in the absence of nuclear effects. We indeed obtain a value close to one over the entire $z$ range as shown in Fig. 5-right since no medium modification effect was included in our simulations. The statistical and systematic errors indicate that in the range $0.1<z<0.5$ variations of $R_{F F}$ larger than $5 \%$ could be measured in both setups. We have also considered the case in which hadrons from jet events are quenched by a factor 5 as observed at RHIC. In this case, the systematic error is under $5 \%$ for both setups. However, the measurement of the nuclear modification factor with an accuracy better than $5 \%$ is prevented by the expected statistics.

We still may consider another measurement approach in which EMCal is employed for prompt photon detection and jets are detected by the central tracking system ${ }^{6}$. In this setup, if similar prompt photon identification features and similar background of PHOS and EMCal are assumed, the prompt photon statistics is enhanced by a factor seven and consequently the statistical errors are reduced by a factor 2.6 .

\section{Photon-tagged hadrons}

Arleo et al. find in Ref [13] under which kinematic conditions the $\gamma-\pi^{0}$ momentum-imbalance distributions may reflect the quark fragmentation function into the pion. In this section we summa-

\footnotetext{
${ }^{6}$ It is not well-advised to use PHOS as a detector of jet neutral particles due to its reduced acceptance.
} 

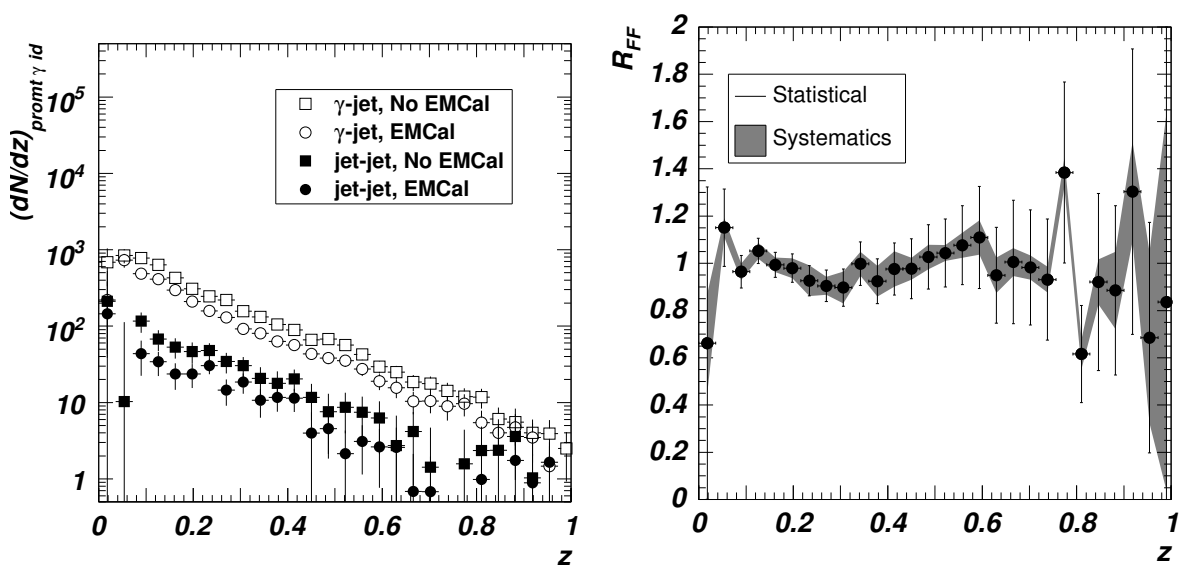

Figure 5: Left frame: Fragmentation function for $\gamma$-jet and jet-jet events with energy larger than $20 \mathrm{GeV}$ for $\mathrm{Pb}-\mathrm{Pb}$ collisions, for a LHC running year, not normalized by the number of collisions. Prompt photons in coincidence with jets were identified in PHOS by medium purity SSA and ICM. The HIC background has been statistically subtracted. Statistical errors are shown in the figure. Right Frame: Ratio of the experimental fragmentation functions of $\gamma$-tagged jets with energy larger than $20 \mathrm{GeV}$ for $\mathrm{Pb}$ - $\mathrm{Pb}$ collisions to $p p$ collisions detected in the central tracking system and EMCal. The shaded region represents the systematic error due to the contamination from jet-jet events. A similar ratio and systematics is obtained without EMCal.

rize which are the most suitable kinematic cuts for the LHC and we show a simple case study at the LHC.

\subsection{Kinematic requirements}

\section{(i) A truly perturbative pion}

In order to construct meaningful $\gamma-\pi^{0}$ correlations, the pion needs to be produced perturbatively, and not from the medium with mechanisms like recombination (at RHIC in the $p_{\perp_{\pi}} \simeq 2-5 \mathrm{GeV} / c$ range [21]). It could be wise to require the pion momentum cut, $p_{\perp \pi}^{\text {cut }}$, to be greater than $10 \mathrm{GeV} / c$ at the LHC.

(ii) A wide $z_{\gamma \pi}$ range

If there is any matching between experimental and theoretical imbalance distributions, the window at which this occurs should of course be as large as possible, i.e. $z_{\min } \lesssim z_{\gamma \pi} \lesssim 1$, with $z_{\text {min }} \ll 1$. We should select as asymmetric as possible $p_{\perp_{\gamma}}^{\text {cut }}$ and $p_{\perp_{\pi}}^{\text {cut }}$.

\section{(iii) A prompt photon}

As already discussed, we can reduce the prompt photon background with isolation cuts, nevertheless, we should play in regions where the prompt photon production is large enough compared to the background. We have seen that with $p_{\perp_{\gamma}}^{\text {cut }} \gtrsim 20 \mathrm{GeV} / c$ we can perform the isolation cut with good results. 


\section{(iv) Reasonable counting rates}

Last but not least, the number of events should remain large enough so that the imbalance distributions can be measured with a high-enough statistical accuracy. Its precise value depends of course crucially on the integrated luminosity. In Fig. 1, we see that with PHOS we can measure with reasonable counting rate prompt photons below $40 \mathrm{GeV}$ and with EMCal, due to its larger acceptance, it can be extended up to about $60 \mathrm{GeV} / c$.

\subsection{Case study at the LHC}

In order to illustrate the above requirements, the $\gamma-\pi^{0}$ imbalance distributions are computed perturbatively in $\mathrm{PbPb}$ collisions at the LHC. The calculations are carried out to LO in $\alpha_{s}$, using the standard fragmentation functions in $p p$ reactions and the rescaled fragmentation functions in central $\mathrm{Pb}-\mathrm{Pb}$ collisions [22] using the BDMPS quenching weight [23] (see [8] for details). The following cuts are chosen:

Case A: $p_{\perp_{\pi}}^{\text {cut }}=10 \mathrm{GeV} / c / p_{\perp_{\gamma}}^{\text {cut }}=20 \mathrm{GeV} / c \quad$ Case B: $p_{\perp_{\pi}}^{\text {cut }}=10 \mathrm{GeV} / c / p_{\perp_{\gamma}}^{\text {cut }}=50 \mathrm{GeV} / c$ Clearly, Case A has the main advantage to offer higher rates than Case B. On the contrary, the $\pi / \gamma$ cuts are not too asymmetric ( $p_{\perp_{\pi}}^{\text {cut }} / p_{\perp_{\gamma}}^{\text {cut }}=1 / 2$ ), and the photons could be abundantly produced through fragmentation because of the not too large cut, $p_{\perp \gamma}^{\text {cut }}=20 \mathrm{GeV} / c$.
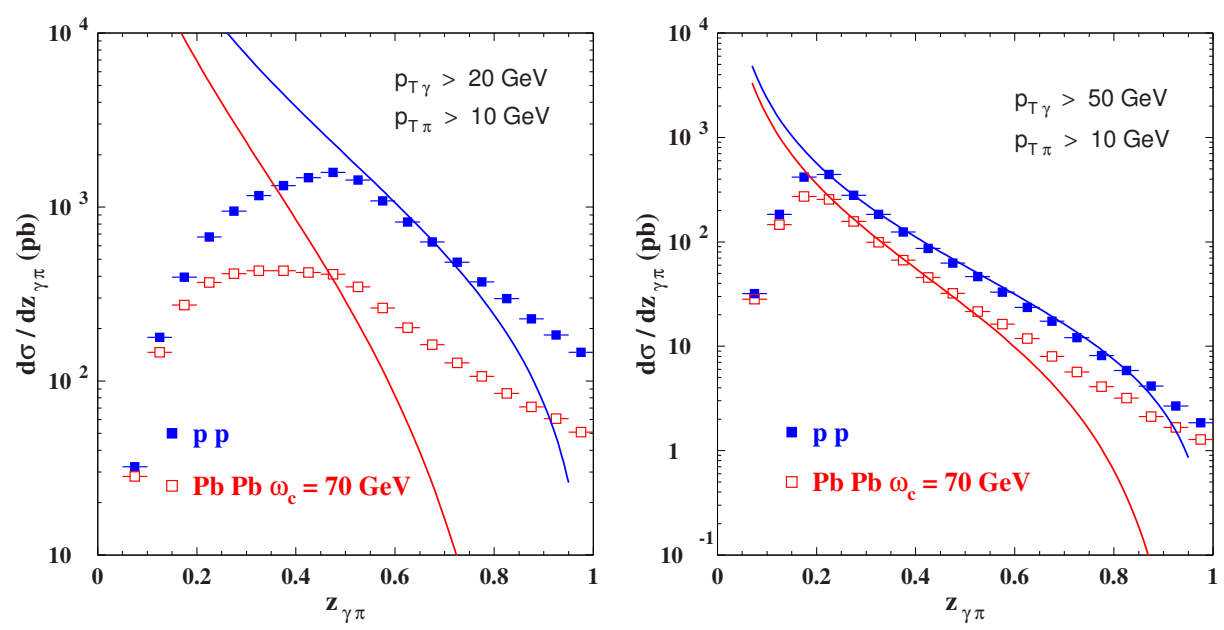

Figure 6: $\gamma-\pi^{0}$ imbalance distributions in Case A (left) and B (right). The solid lines show the (rescaled) quark fragmentation functions used in the calculation. $\mathrm{Pb}-\mathrm{Pb}$ collisions were considered with jet-quenching being $\omega_{C}$ the scale of the energy loss defined as $1 / 2 \hat{q} L^{2}$, where $\hat{q}$ is the coefficient transport and $L$ is the medium path length [5].

The $\gamma-\pi^{0}$ imbalance distributions in $p p$ and $\mathrm{PbPb}$ collisions are plotted in Figure 6 for the Case A (Left) and B (right). In order to show the possible connection between $\mathrm{d} \sigma / \mathrm{d} z_{\gamma \pi}$ and the theoretical fragmentation function, the "input" vacuum and medium-modified quark fragmentation into the pion used in the calculation (arbitrarily rescaled so as to match $p p$ ) are also displayed. When $p_{\perp_{\gamma}}^{\text {cut }}=20 \mathrm{GeV} / c$, there is absolutely no matching between those two quantities. This is because of the important contribution of the fragmentation-photon channel, as well as the restricted kinematic $z_{\gamma \pi}$ window, $0.5 \leq z_{\gamma \pi} \leq 1$, over which imbalance distributions and fragmentation functions could have matched. On the contrary, a much better "agreement" is found in Case 
$\mathrm{B}$, from $z_{\gamma \pi} \simeq p_{\perp_{\pi}}^{\text {cut }} / p_{\perp_{\gamma}}^{\text {cut }}=0.2$ up to $z_{\gamma \pi} \simeq 0.9$ in $p p$ and $z_{\gamma \pi} \simeq 0.6$ in PbP collisions, over which the fragmentation-photon channel becomes predominant.

Taking into account the counting rates in EMCal and PHOS, it seems that $p_{\perp_{\gamma}}^{\text {cut }}=40-50 \mathrm{GeV} / c$ proves by far more advantageous than the lower cut $p_{\perp_{\gamma}}^{\text {cut }}=20 \mathrm{GeV} / c$, which clearly misses the main interest of photon-tagged hadrons: probing vacuum and medium-modified fragmentation functions. It is possible that if we decrease $p_{\perp \pi}^{\text {cut }}$, we could obtain better results at $p_{\perp_{\gamma}}^{\text {cut }}=20 \mathrm{GeV} / c$.

\section{Conclusions}

We developed an algorithm to identify prompt photons and $\gamma$-jet events generated in $p p$ and $\mathrm{Pb}-\mathrm{Pb}$ collisions in ALICE. Prompt photons are identified efficiently in PHOS, and possibly by EMCAL, with the help of a shower shape analysis, which is capable of rejecting hadrons, and the isolation cut criterion to reject $\pi^{0}$ mesons. We estimated the spectrum of the identified prompt photons for the statistics of the ALICE integrated luminosity in a standard year of running at LHC. Photon-jet events were identified by selecting a prompt photon in PHOS and searching for a leading particle in the opposite direction inside the ALICE central tracking system. Jets were reconstructed by an algorithm which takes all particles within a cone around the leading particle found which has to fulfill the requirement of being correlated with the photon. As jet-jet events have a larger cross section than photon-jet events, they originate a considerable background due to $\pi^{o}$ decay photons misidentified in PHOS as direct photons. In the configuration with EMCal, these events are effectively rejected and their contribution reduced to a negligible level of contamination by shower shape and isolation cut analysisy [12]. Fragmentation functions can be accurately calculated and used to obtain the nuclear modification factor, $R_{F F}$. We found that nuclear medium modifications can be measured if they produce variations of $R_{F F}$ larger than $5 \%$ in the region $0.1<z<0.5$. If we use EMCAL as prompt photon detector, the same conclusions may be achieved, increasing the reach in $p_{T}$ for the prompt photon detection. The prompt gamma-hadron correlations are also a promising tool to study medium modifications, selecting a $p_{T}$ cut on the hadron of $10 \mathrm{GeV} / \mathrm{c}$ and of $50 \mathrm{GeV} / c$ on the photon.

\section{Acknowledgments}

I am grateful for the support, comments and advices of H. Delagrange, J. Díaz, Y.V. Kharlov and specially Y. Schutz, without them this work would not have been possible. It is also a pleasure to thank F. Arleo for giving me the opportunity to show his work on gamma-hadron correlations. I would like to thank to the organizers for inviting me to this very interesting workshop and to the ALICE group in Frascati for sending me there.

\section{References}

[1] A. Accardi et al, hep-ph/0310274 (2004)

[2] J. D.Bjorken, FERMILAB-PUB-82-059-THY (1982)

[3] C. Adler et al., Phys. Rev. Lett. 89, 0202301 (2002)

[4] S. Adler et al., Phys. Rev. Lett. 91, 072301 (2003) 
[5] C. A. Salgado and U. A.Wiedemann, Phys. Rev. Lett. 93, 42301 (2004)

[6] F. Arleo et al., JHEP 11, 009 (2004)

[7] X-N. Wang. et al., Phys. Rev. C 55, 3047 (1997)

[8] F. Arleo et al., JHEP 11 (2004) 009 [hep-ph/ 0410088 ;

Arleo F, JHEP 09 (2006) 015 [hep-ph / 0601075 ].

[9] G. Conesa et al., Nucl. Instr. and Meth. A 537, 363-367 (2005)

[10] T. Cormier, et al., ALICE Collaboration, The Electromagnetic Calorimeter. Addendum to the ALICE Technical Proposal, CERN-LHCC-2006-014, CERN-LHCC-96-32-ADD-3, 02 March 2006

[11] T. Cormier et al., Eur. Phys. J. C 34 (2004) s1.333-s1.345.

[12] G. Conesa et al., ALICE-INT-2005-014, (2005)

[13] F. Arleo, Proceedings of Quark Matter 2006; Photon-tagged correlation in heavy-ion collisions: kinematic requirements and case study, [hep-ph/0701207].

[14] ALICE Collaboration, J. Phys. G: Nucl. Part. Phys. 30, 1517-1763 (2004)

[15] T. Sjostrand et al., [hep-ph/0108264], (2001)

[16] M. Gluck, Z. Phys. C 67, 433-448 (1995)

[17] M. Gyulassy and X-N. Wang, Comput. Phys. Commun. 83, 307-331 (1994)

[18] F. Arleo et al., Photon physics section, CERN Yellow Report 2004-009 [hep-ph/ 0311131$].$

[19] ALICE Collaboration, J. Phys. G: Nucl. Part. Phys. 32 (2006) 1295-2040

[20] K. Adcox et al., Nucl. Phys. A757 (2005) 184-283 [nucl-ex/ 0410003 ];

J. Adams et al., Nucl. Phys. A757 (2005) 102-183 [nucl-ex/0501009].

[21] R. J. Fries et al., Phys. Rev. Lett. 90 (2003) 202303 [nucl-th/ 0301087 ].

[22] X-N. Wang et al., Phys. Rev. Lett. 77 (1996) 231-234 [hep-ph/9605213].

[23] F. Arleo, JHEP 11 (2002) 044 [hep-ph / 0210104 ]. 